\title{
Das Ende der bilateralen Handelsabkommen?
}

Was Mega-Abkommen wie das Freihandelsabkommen EU-USA (TTIP) für die

Schweiz bedeuten

Charlotte Sieber-Gasser *

Zwei Jahrzehnte lang hat sich im internationalen Handelsrecht wenig bewegt. Durch die Paralyse der WTO genossen bilaterale Handelsabkommen viel Aufmerksamkeit und waren während 20 Jahren das primäre Instrument für die Weiterentwicklung und Vertiefung der Handelsbeziehungen. Vor gut einem Jahr hat sich die Dynamik geändert: Mit mehreren sogenannten Mega-Abkommen, plurilateralen Initiativen und dem ersten neuen Entscheid der WTO seit vielen Jahren, verlieren bilaterale Handelsabkommen an Attraktivität und Nutzen. Diese neue Dynamik bringt deutlich zum Vorschein, dass der handelspolitische Alleingang steigende Kosten mit sich bringt und die fortschreitende Globalisierung einen Imperativ zur Vernetzung und Kooperation schafft. Ob die Schweiz bereit ist, der neuen Herausforderung proaktiv zu begegnen, wird sich weisen.

I. Die neuen Abkommen im globalen Kontext ..................................................2

II. Mega-Abkommen und Kleinstaaten: Das TTIP und die Schweiz ......................... 3

1. Das TTIP und die Handelsbeziehungen Schweiz - EU .................................5

2. Das TTIP und die Handelsbeziehungen Schweiz - USA ................................ 7

3. Die Kosten des Abseitsstehens für Kleinstaaten........................................... 9

III. Neue Herausforderungen in der Aussenwirtschaftspolitik ............................... 12

1. Von Chlorhühnchen und skrupellosen Investoren ........................................ 12

2. Der eigentliche Kern der Herausforderung ............................................. 14

IV. Die Neue Komplexität als Chance ................................................................. 16

Zitiervorschlag: Charlotte Sieber-Gasser, Das Ende der bilateralen Handelsabkommen?, in: sui-generis 2015, S. 1

URL: $\quad$ sui-generis.ch/10

DOI: $\quad$ https://doi.org/10.21257/sg.10

* Dr. Charlotte Sieber-Gasser ist Post-Doctoral Research Fellow am NCCR Trade Regulation und unterrichtet Wirtschaftsvölkerrecht und Recht \& Entwicklung am World Trade Institute und an der Universität Bern.

Mit herzlichem Dank für die engagierten Diskussionen, die differenzierte und ehrliche Kritik sowie die kritische Durchsicht des Manuskripts an Matthias Oesch, Nicolas Diebold, Rachel Liechti, Stefan Schlegel und Daniel Hürlimann.

Dieses Werk ist lizenziert unter einer Creative Commons Namensnennung - Weitergabe unter gleichen Bedingungen 4.0 International Lizenz. 


\section{Die neuen Abkommen im globalen Kontext}

1 Die fortschreitende Globalisierung im Bereich des Handels wurde insbesondere geprägt durch die Errichtung der Welthandelsorganisation (WTO) 1995. Die WTO führte erstmals international geltende und bindende Minimalstandards in allen wesentlichen Bereichen des internationalen Handelsrechts ein. Parallel oder in Ergänzung zu diesen internationalen Minimalstandards entwickelten die WTO-Mitgliedstaaten die Marktzugangsbestimmungen über bilaterale oder regionale Handelsabkommen weiter. ${ }^{1}$ So ist ein komplexes Rechtsgebiet entstanden, geprägt vom Zusammenspiel mehrerer regulatorischer Ebenen, der internationalen Ebene, der regionalen und bilateralen Ebene und schliesslich der unilateralen Ebene, in welcher WTO-Mitgliedstaaten unilateral ihre eigenen Märkte weiter öffnen. Während die WTO bei ihrer Errichtung noch aus 128 Mitgliedstaaten bestand, zählt sie seit dem 26 . Juni 2014160 Mitgliedstaaten $^{2}$ und hat seit bald 20 Jahren keine grösseren Verhandlungserfolge mehr feiern können.3 Der Umfang des Verhandlungsmandats vom Dezember 2013 in Bali war relativ beschränkt, ist allerdings seit einigen Jahren und nur dank erfolgreichen Nachverhandlungen zwischen den USA und Indien erstmals wieder ein substantieller Entscheid, welcher von allen Mit-

Zwischen 1990 und 2010 von 70 auf über 300 Abkommen, siehe WTO, World Trade Report 2011, Genf, 2011, S. 54.

Siehe WTO, Members and Observers.

Abgesehen von Bali im Dezember 2013, wurde der letzte Entscheid 2005 in Hong Kong verabschiedet und hält in erster Linie fest, dass weiter verhandelt werden soll, siehe WTO, Ministerial Declarations and Decisions. gliedstaaten mitgetragen wird. 4

2 Die Pendelbewegung zwischen multilateralen, nicht-diskriminierenden 5 und präferenziellen, diskriminierenden Verhandlungen ${ }^{6}$ konnte in den vergangenen 200 Jahren schon mehrfach beobachtet werden(so z.B. im Güterhandel, im Schutz des geistigen Eigentums oder im Dienstleistungshandel): Ab einer gewissen Anzahl präferenzieller Abkommen weltweit steigt jeweils der Druck, allgemein verbindliche Minimalstandards im Rahmen von multilateralen Verhandlungen zu vereinbaren (siehe Spaghetti-Bowl Effekt). 7 Stark vereinfacht kann diese Dynamik wie folgt umschrieben werden: Handelsbeziehungen werden zunehmend komplexer und umfassen neue Rechtsge-

4 Die Verhandlungen mit Indien liefen bis im November 2014. Nur knapp konnte ein Scheitern des Bali-Entscheids verhindert werden. Siehe z.B. Erasmus, Gerhard, The WTO Breakthrough on the Bali Package: The Trade Facilitation Value Chain and African Integration, Tralac, 2014.

5 Multilaterale Verhandlungen sind Verhandlungen innerhalb einer internationalen Organisation (wie früher dem GATT und heute der WTO) über allgemein verbindliche Standards, welche für alle Mitglieder der Organisation gelten und neuen Mitgliedern diskriminierungsfrei offenstehen. Der Zweck von multilateralen Verhandlungen ist insbesondere, dass sich möglichst viele Staaten anschliessen.

6 Präferenzielle Verhandlungen sind Verhandlungen zwischen zwei oder mehr Staaten, welche sich ausschliessliche Präferenzen gewähren, und deshalb gegenüber Drittstaaten sogenannt diskriminierend wirken. Der Zweck von präferenziellen Verhandlungen ist üblicherweise gerade, dass Drittstaaten von den verhandelten Vorteilen ausgeschlossen bleiben.

7 Siehe Cottier, Thomas, Sieber-Gasser, Charlotte und Wermelinger, Gabriela, The Dialectical Relationship of Preferentialism and Multilateralism, in: M. Elsig and A. Dür (eds.), Trade Cooperation: The Purpose, Design and Effects of Preferential Trade Agreements, Cambridge University Press, Cambridge, 2015; Cottier, Thomas, International Economic Law in Transition from Trade Liberalisation to Trade Regulation, Journal of International Economic Law, vol. 17, nr. 3, 2014, S. 671-677, S. 275. 
biete, was dazu führt, dass die multilateral geltenden Minimalstandards nicht mehr ausreichen, um allen Herausforderungen des Handels zu begegnen. In bilateralen und regionalen Handelsabkommen werden Handelsbeziehungen rechtlich modernisiert und an die aktuellen Entwicklungen angepasst. Je zahlreicher solche Abkommen, desto unübersichtlicher werden die geltenden handelsrechtlichen Bestimmungen im Einzelfall. Dies schafft genügend Druck, damit auf internationaler Ebene wieder neue Abkommen abgeschlossen werden können, welche neue Minimalstandards festlegen.

3 Die neusten Entwicklungen im internationalen Handelsrecht unterscheiden sich aber in einem wesentlichen Aspekt von der vergangenen Dynamik zwischen Multilateralismus und Bilateralismus: Anstatt immer mehr regionaler und bilateraler Initiativen, schliessen sich neu grosse Gruppen von Ländern und regionale Märkte in sogenannten MegaAbkommen zusammen: ${ }^{8}$ Zum Multilateralismus und Bilateralismus ist mit den Mega-Abkommen neu ein Instrument hinzugestossen, welches auch mit Minilateralismus ${ }^{9}$ oder Plurilateralismus ${ }^{10}$ umschrieben wird. Präferenzielle Verhandlungen zwischen mehreren Ländern, welche eine grosse geografische Fläche abdecken und gemeinsam über einen sub-

8 Baghwati, Jagdish, Pravin, Krishna und Panagriya, Arvind, The World Trade System: Trends and Challenges, 2014, S. 3.

9 Siehe z.B. auch Brummer, Chris, Minilateralism: How Trade Alliances, Soft Law, and Financial Engineering are Redefining Economic Statecraft, Cambridge University Press, New York, 2014.

10 Plurilateralismus bezeichnet im Kontext des Handelsrechts Abkommen, welche allen WTOMitgliedstaaten auf freiwilliger Basis offen stehen, aber nicht alle Mitgliedstaaten umfassen: WTO Glossary. stantiellen Anteil am Weltmarkt verfügen. Dies sind Abkommen zwischen zwei oder mehr Weltregionen (z.B. Transpazifik (TPP) und Transatlantik (TTIP und CETA)) ${ }^{11}$ oder Abkommen mit mehr als 40 Mitgliedstaaten (z.B. öffentliches Beschaffungswesen (GPA) oder Dienstleistungen (TiSA) $)^{12}$, welche potentiell den Weltmarkt auf einer thematischen oder geografischen Achse dominieren werden.

4 Die Schweiz ist als WTO-Mitglied und globalisierte Volkswirtschaft ebenfalls Teil dieser neuen Dynamik und einer der Mitgliedstaaten, welche das TiSAAbkommen verhandeln. ${ }^{13}$ An den weiteren neueren minilateralen Initiativen nimmt die Schweiz allerdings aktuell nicht teil.

\section{Mega-Abkommen und Kleinstaa- ten: Das TTIP und die Schweiz}

5 Die neue Bewegung im internationalen Handelsrecht betrifft die Schweiz direkt im Rahmen der WTO-Mitgliedschaft und als Verhandlungspartnerin im TiSA. Sie

11 So aktuell das Transpazifische Partnerschaftsabkommen (Transpacific Partnership Agreement, TPP), welches mind. 11 Länder rund um den Pazifik umfasst und etwa 30 Prozent des Welt-BIPs; die transatlantische Handels- und Investitionspartnerschaft (Transatlantic Trade and Investment Partnership, TTIP) zwischen der EU und den USA, welche etwa 40 Prozent des Welt-BIPs umfasst; und das Wirtschafts- und Handelsabkommen zwischen Kanada und der EU (Comprehensive Economic and Trade Agreement, CETA), welches etwa 20 Prozent des Welt-BIPs umfasst.

12 So z.B. das Übereinkommen über das öffentliche Beschaffungswesen (General Procurement Agreement, GPA), welches bald 53 Länder umfasst; oder das Abkommen über den Handel mit Dienstleistungen (Trade in Services Agreement, TiSA), welches aktuell 50 Länder umfasst und 70 Prozent des Handels mit Dienstleistungen weltweit.

13 Siehe Staatssekretariat für Wirtschaft Seco, Die Schweiz und das TiSA, 2014. 
betrifft die Schweiz aber vor allem auch indirekt, indem sich ihre unmittelbaren Nachbarländer oder wichtige Exportmärkte den neuen Mega-Abkommen anschliessen. Die Auswirkungen des CETAAbkommens zwischen der EU und Kanada mögen für die Schweiz insgesamt geringfügig sein, insbesondere weil sie bereits über ein Freihandelsabkommen und ein Abkommen über die gegenseitige Anerkennung von Standards mit Kanada verfügt. ${ }^{14}$ Obwohl das CETA-Abkommen bedeutend umfassender ist, als das Freihandelsabkommen Kanada - Schweiz, wird der zu erwartende Wettbewerbsvorteil der Europäer in Kanada bis zu einem gewissen Grad mit dem bestehenden privilegierten Marktzugang der Schweiz abgefedert. Zudem ist der kanadische Markt nicht gleich bedeutsam für das Schweizer Exportvolumen, wie dies der europäische und der amerikanische Markt sind. Möglich bleibt, dass das CETA-Abkommen die Schweizer Exportwirtschaft in einzelnen Aspekten vor Herausforderungen stellen wird. Sollte das geplante TTIP Freihandelsabkommen zwischen der EU und den USA zustande kommen, dürften die Auswirkungen auf die Schweiz aber auf jeden Fall spürbar sein.

6 Bilaterale Handelsabkommen müssen, damit das Meistbegünstigungsprinzip der WTO15 nicht zur Anwendung kommt und somit der präferenzielle Charakter des Abkommens verloren gehen würde, umfassend sein: GATT Art. XXIV und

14 Staatssekretariat für Wirtschaft Seco, Freihandelsabkommen EFTA - Kanada.

15 Das Meistbegünstigungsprinzip sorgt dafür, dass Präferenzen, welche einem Mitglied gewährt werden automatisch auch auf alle anderen WTOMitglieder angewendet werden sollen. WTO, Principles of the Trading System, 2014.
GATS Art. V lassen Ausnahmen vom Meistbegünstigungsprinzip zu, wenn ein Abkommen grundsätzlich allen Handel zwischen den Vertragsparteien umfasst und grundsätzlich alle Handelshemmnisse beseitigt. ${ }^{16}$ Von dieser Regelung sind auch die neuen Mega-Abkommen nicht ausgenommen. Das bedeutet, dass diese neuen Abkommen umfassend sein müssen und die Märkte weitgehend öffnen müssen. Es bedeutet aber auch, dass Drittstaaten wie die Schweiz von dieser Öffnung ausgeschlossen bleiben. Eine Ausnahmeregelung vom Meistbegünstigungsprinzip besteht nur für den Handel mit Waren und Dienstleistungen. Die anderen WTO-Abkommen sehen keine solche Ausnahme vor. Demzufolge würde zum Beispiel Marktöffnung im Bereich der technischen Handelshemmnisse und des geistigen Eigentums im Rahmen eines Mega-Abkommens wie dem TTIP auch für Drittstaaten offen stehen können. ${ }^{17}$

7 Entgegen des ambitiösen Verhandlungsmandats über das TTIPAbkommen, wird allgemein davon ausgegangen, dass das Freihandelsabkommen zwischen der EU und den USA Zölle zwischen den beiden Märkten abschaffen wird, jedoch vorerst die Harmonisierung

16 Detailliert, siehe Islam, Md. Rizwanul und Alam, Shawkat, Preferential Trade Agreements and the Scope of GATT Article XXIV, GATS Article V and the Enabling Clause: An Appraisal of GATT/WTO Jurisprudence, Netherlands International Law Review, vol. 56, no. 1, 2009, S. 1-34.

17 Siehe dazu auch Horn, Henrik, Mavroidis, Petros C. und Sapir, André, Beyond the WTO? An Anatomy of EU and US Preferential Trade Agreements, The World Economy, vol. 33, no. 11, 2010, S. 15651588; und insbesondere Bartels, Lorand, The Legality of the EC Mutual Recognition Clause under WTO Law, Journal of International Economic Law, vol. 8, no. 3, 2005, S. 691-720. 
und gegenseitige Anerkennung des Rechts (die sog. nicht-tarifären Handelshemmnisse oder auch «behind the border barriers to trade») nur in einem geringen Umfang erreichen kann. ${ }^{18}$ Nach einer Studie des World Trade Institutes im Auftrag des Staatssekretariats für Wirtschaft (SECO) entspricht dies dem für die Schweiz mit den grössten Nachteilen verbundenen Szenario. ${ }^{19}$ Die möglichen Auswirkungen auf die Schweiz im Detail verbleiben angesichts der noch laufenden Verhandlungen unklar. Weil sowohl der europäische wie auch der amerikanische Markt für die Schweiz grosse Bedeutung haben, sind dennoch bereits heute einzelne Tendenzen vorhersehbar. Sie sind je nach Ausgestaltung der Marktöffnung, sowie abhängig vom jeweiligen Exportmarkt unterschiedlich wegen den unterschiedlichen handelsrechtlichen Beziehungen zwischen der Schweiz und der EU und der Schweiz und den USA.

\section{Das TTIP und die Handelsbezie- hungen Schweiz - EU}

8 Die Handelsbeziehungen der Schweiz mit der EU stützen sich heute primär auf das

18 Siehe z.B. Baghwati et al., The World Trade System: Trends and Challenges, 2014, S. 22; Pardo, Romain, The Transatlantic Trade and Investment Partnership: A Long Hard Road to Multilateralism?, EPC Commentary, 2013; Health Concerns raised over EU-US Trade Deal, The Lancet, vol. 384, nr. 9946, 2014, S. 843-844, S. 844; Egger, Peter, Francois, Joseph, Manchin, Miriam und Nelson, Douglas, 'Non-Tariff Barriers, Integration, and the Trans-Atlantic Economy', Economic Policy, vol. 30, nr. 81, 2015.

19 Siehe Cottier, Thomas, Egger, Peter, Francois, Joseph, Manchin, Miriam, Shingal, Anirudh und Sieber-Gasser, Charlotte, Potential Impacts of a EU-US Free Trade Agreement on the Swiss Economy and External Economic Relations, Gutachten zuhanden Staatssekretariat für Wirtschaft (SECO), 2014, S. 37ff; siehe auch Egger et al., 2015.
Freihandelsabkommen Schweiz-EU von $1972^{20}$, welches mit dem Güterverkehrsabkommen und den Bilateralen I und II sowie Schengen/Dublin ergänzt wird im Sinne einer umfassenden gegenseitigen Marktöffnung und -integration. Das Abkommen schafft Zölle sowie Kontingente für Industriegüter ab und verbietet nichttarifäre Handelshemmnisse mit gleicher Wirkung. ${ }^{21}$ Es umfasst keine Landwirtschaftsprodukte, während bei den landwirtschaftlichen Verarbeitungsprodukten nur der industrielle Verarbeitungsteil zollfrei ist. ${ }^{22} 1990$ wurde das Freihandelsabkommen ergänzt mit dem Güterverkehrsabkommen, welches die Zollabfertigung vereinfacht. ${ }^{23}$ Im engeren Sinn der gegenseitigen Marktöffnung und somit direkt auf die Regulierung des Marktzugangs anwendbar sind weitere Abkommen der Bilateralen I, welche Handelshemmnisse abbauen: Das Abkommen über den Abbau technischer Handelshemmnisse ${ }^{24}$, das Abkommen über das öffentliche Beschaffungswesen $^{25}$, das Land-26 und das Luftverkehrs-

20 Abkommen zwischen der Schweizerischen Eidgenossenschaft und der Europäischen Wirtschaftsgemeinschaft, 22. Juli 1972, SR 0.632.401.

21 FHA Schweiz-EU, Art. 18.

22 Protokoll Nr. 2 zum FHA Schweiz-EU.

23 Abkommen vom 21. November 1990 zwischen der Schweizerischen Eidgenossenschaft und der Europäischen Wirtschaftsgemeinschaft über die Erleichterung der Kontrollen und Formalitäten im Güterverkehr, 21. November 1990, SR 0.631.242.05. Ersetzt durch das Abkommen zwischen der Schweizerischen Eidgenossenschaft und der Europäischen Gemeinschaft über die Erleichterung der Kontrollen und Formalitäten im Güterverkehr und über zollrechtliche Sicherheitsmassnahmen, 25. Juni 2009, SR 0.631.242.05.

24 Abkommen zwischen der Schweizerischen Eidgenossenschaft und der Europäischen Gemeinschaft über die gegenseitige Anerkennung von Konformitätsbewertungen, 21. Juni 1999, SR 0.946.526.81.

25 Abkommen zwischen der Schweizerischen Eidgenossenschaft und der Europäischen Gemeinschaft 
abkommen ${ }^{27}$ sowie das Landwirtschaftsabkommen ${ }^{28}$. Im Rahmen der Bilateralen II wurde unter anderem das Abkommen über landwirtschaftliche Verarbeitungserzeugnisse abgeschlossen ${ }^{29}$, welches den Zugang zum europäischen Raum über das Freihandelsabkommen von 1972 hinaus erleichtert. Ein Spezialfall stellt das Abkommen über die Personenfreizügigkeit3o dar: Während hier nicht primär Handelserleichterungen im Zentrum stehen, sondern die gegenseitige Marktöffnung und -integration, so ist es neben den Land- und Luftverkehrsabkommen das einzige Abkommen zwischen der Schweiz und der EU, welches am Rande auch den Handel mit Dienstleistungen umfasst. Alles in allem verfügt die Schweiz heute über einen komplex geregelten weitgehend von Handelshemmnissen befreiten Zugang zum europäischen Markt, mit Ausnahme der Dienst-

über bestimmte Aspekte des öffentlichen Beschaffungswesens, 21. Juni 1999, SR 0.172.052.68.

26 Abkommen zwischen der Schweizerischen Eidgenossenschaft und der Europäischen Gemeinschaft über den Güter- und Personenverkehr auf Schiene und Strasse, 21. Juni 1999, SR 0.740.72.

27 Abkommen zwischen der Schweizerischen Eidgenossenschaft und der Europäischen Gemeinschaft über den Luftverkehr, 21. Juni 1999, SR 0.748.127.192.68.

28 Abkommen zwischen der Schweizerischen Eidgenossenschaft und der Europäischen Gemeinschaft über den Handel mit landwirtschaftlichen Erzeugnissen, 21. Juni 1999, SR 0.916.026.81.

29 Abkommen zwischen der Schweizerischen Eidgenossenschaft und der Europäischen Gemeinschaft zur Änderung des Abkommens zwischen der Schweizerischen Eidgenossenschaft und der Europäischen Wirtschaftsgemeinschaft vom 22. Juli 1972 in Bezug auf die Bestimmungen über die landwirtschaftlichen Verarbeitungserzeugnisse, 26. Oktober 2004, SR 0.632.401.23.

30 Abkommen zwischen der Schweizerischen Eidgenossenschaft einerseits und der Europäischen Gemeinschaft und ihren Mitgliedstaaten andererseits über die Freizügigkeit, 21. Juni 1999,

SR 0.142.112.681. leistungen und der Agrarprodukte.

9 Die Stellung der Schweizer Anbieter von Produkten und Dienstleistungen im europäischen Markt würde durch das TTIPAbkommen geschwächt werden. Der europäische Markt ist für die Schweiz der wichtigste: Rund 55\% der Schweizer Exporte gehen in die EU Mitgliedstaaten. ${ }^{31}$ Bisher wurden Schweizer Industrieprodukte - nicht aber Dienstleistungen (abgesehen vom GATS-Minimalstandard) und verarbeitete Landwirtschaftsprodukte sowie Agrarprodukte - gleichbehandelt mit den Produkten aus den EU Mitgliedstaaten. Auch im öffentlichen Beschaffungswesen verfügt die Schweiz über einen weitgehend privilegierten Marktzugang. ${ }^{2}$

10 Es ist davon auszugehen, dass ein TTIPAbkommen den Export von Industrieprodukten aus den USA in den europäischen Markt weitgehend liberalisiert. Das bedeutet, dass Schweizer Industrieprodukte plötzlich verstärkte Konkurrenz aus den USA erhalten. Je nach Sektor und Produkt mögen dann die USProduzenten in Europa über einen Wettbewerbsvorteil gegenüber den Schweizer Produzenten verfügen, da es ihnen möglich ist, ihre Produkte zu günstigeren Konditionen auf den europäischen Markt zu bringen.33 Dies ist insbesondere der Fall für den Export von Gütern welche heute noch mit Zoll belastet sind und allein durch den Wegfall des Zolls deutlich

31 Eidgenössisches Departement für auswärtige Angelegenheiten, Switzerland and the European Union, Bern, 2014, S. 22.

32 Siehe Abkommen über bestimmte Aspekte des öffentlichen Beschaffungswesens, SR 0.172.052.68.

33 Nach China sind die USA das aktuell wettbewerbsfähigste Land in Bezug auf Produktionskosten, siehe Finanzen.at, 2014. 
günstiger angeboten werden könnten. 34 Wegen den fehlenden Abkommen im Bereich der Dienstleistungen und der verarbeiteten Landwirtschaftsprodukten und Agrarprodukten, erhalten möglicherweise amerikanische Anbieter in diesen Bereichen privilegierteren Zugang zum europäischen Markt. In diesen Bereichen dürfte der Schweizer Export ebenfalls von einem verstärkten Wettbewerb betroffen sein.

11 Während die Schweiz bereits über verschiedene Abkommen zur gegenseitigen Anerkennung von Produktionsstandards mit der EU verfügt, ist es das erklärte Ziel der Verhandlungspartner im TTIPAbkommen, ebendiese Standards schrittweise $\mathrm{zu}$ harmonisieren. Bereits heute verfügen die USA und die EU über einzelne Abkommen zur gegenseitigen Anerkennung von Produktionsstandards, so z.B. im Bereich der Standards für Produkte aus biologischem Anbau. Diese Standards stimmen nicht mit denjenigen überein, welche die Schweiz mit der EU abgeschlossen hat.35 In der Folge richten sich die Produzenten in der EU lieber nach den Bestimmungen der USA, weil der Markt dort deutlich grösser ist. ${ }^{6}$ Das bedeutet für Schweizer Produzenten von Bio-Produkten, dass sie einzelne Bestandteile nicht mehr in der EU einkaufen können, weil sie dadurch das Recht verlieren würden, ihr Produkt unter dem Label ,Bio’ zu verkaufen. Gerade solche

34 Siehe dazu auch Francois, Joseph und SieberGasser, Charlotte, Zum Freihandelsabkommen EUUSA: Auswirkungen auf die Schweiz, SwissTnet, inSide online, 2014.

35 Siehe Alimenta, Die Bio-Schweiz bleibt aussen vor, Bern, 2012.

362010 war der Umsatz mit Bio-Lebensmitteln in den USA rund 24.8 Milliarden USD; 2013 war der Umsatz mit Bio-Lebensmitteln in der Schweiz rund 2.05 Milliarden CHF.
Beispiele könnten sich häufen, sollten sich die EU und die USA über die Harmonisierung und Anpassung von Produktionsstandards einig werden. Ein Abseitsstehen hat für die Schweiz nicht nur zur Folge, dass ihr Exportvolumen in die beiden Märkte EU und USA unter Druck gerät, sondern, dass möglicherweise bereits die Produktion in der Schweiz mit Schwierigkeiten konfrontiert wird. 37

12 Ein einfacher Weg für betroffene Firmen, diesen Schwierigkeiten zu begegnen, ist der Wegzug ins europäische Ausland. Mit der Verlegung der Produktion und allenfalls auch des Firmensitzes in einen EUMitgliedstaat kommen Schweizer Produzenten in den Genuss des vollen Zugangs zum europäischen Binnenmarkt und des privilegierten Zugangs zum amerikanischen Markt. Je nach Umfang des TTIPAbkommens und der Bestimmungen in den einzelnen Sektoren könnte der wirtschaftliche Vorteil einer Verlegung ins europäische Ausland so gewichtig sein, dass er die Gründe, welche bisher gegen eine Verlegung gesprochen haben, weitgehend aufwiegt.

\section{Das TTIP und die Handelsbezie- hungen Schweiz - USA}

13 Im Verhältnis zu den USA bauen die Handelsbeziehungen der Schweiz primär auf das WTO-Recht. Von Verhandlungen über ein Freihandelsabkommen wurde nach exploratorischen Gesprächen 2006 aus verschiedenen Gründen abgesehen. $3^{8}$

37 Hinzu kommt auch der Aspekt der Ursprungsregeln: Sollten diese präferenziell und streng ausgestaltet sein, dürften insbesondere die Schweizer Exporte an Zwischenprodukten, Bau- und Bestandteilen unter Druck geraten, siehe dazu Francois und Sieber-Gasser, 2014.

38 Siehe NZZ, Freihandelsabkommen mit den USA noch nicht spruchreif, 2006; Nell, Philippe G. und 
In den Handelsbeziehungen Schweiz USA gelten also die internationalen Minimalstandards der grossen WTOHandelsabkommen GATT39, GATS 40 , TRIPS41, TBT42 und SPS43 (nebst weiteren Abkommen). 44 Die multilateralen Abkommen werden zwischen den USA und der Schweiz mit den plurilateralen Bestimmungen aus dem GPA45, dem Abkommen über das Beschaffungswesen, komplementiert. All diese Abkommen schaffen eine umfassende Regelung der Minimalstandards im Handel zwischen den USA und der Schweiz. Jedoch ist der Schweizer Export im amerikanischen Markt gleichgestellt mit sämtlichen anderen Anbietern aus Drittstaaten, verfügt also nicht über einen privilegierten Marktzugang. Aus den gescheiterten

Zimmermann, Thomas A., Wege zur Intensivierung der Wirtschaftsbeziehungen Schweiz-USA, Die Volkswirtschaft, 7/8-2007, 2007, S. 43-46, S. 44.

39 Allgemeines Zoll- und Handelsabkommen (GATT) 1994, SR 0.632.21.

40 Allgemeines Abkommen über den Handel mit Dienstleistungen (GATS), Anhang 1.B des Abkommens zur Errichtung der Welthandelsorganisation (WTO), 15. April 1994, SR 0.632.20.

41 Abkommen über handelsbezogene Aspekte der Rechte an geistigem Eigentum (TRIPS), Anhang 1.C des Abkommens zur Errichtung der Welthandelsorganisation (WTO), 15. April 1994, SR 0.632.20.

42 Übereinkommen über technische Handelshemmnisse, Anhang 1A.6 des Abkommens zur Errichtung der Welthandelsorganisation (WTO), 15. April 1994, SR 0.632.20.

43 Übereinkommen über die Anwendung gesundheitspolizeilicher und pflanzenschutzrechtlicher Massnahmen, Anhang 1A.4 des Abkommens zur Errichtung der Welthandelsorganisation (WTO), 15. April 1994, SR 0.632.20.

44 Beispielsweise das Übereinkommen über die Landwirtschaft, das Übereinkommen über Textilwaren und Bekleidung oder das Übereinkommen über handelsbezogene Investitionsmassnahmen, Anhang des Abkommens zur Errichtung der Welthandelsorganisation (WTO), 15. April 1994, SR 0.632.20.

45 Übereinkommen über das öffentliche Beschaffungswesen, 15. April 1994, SR 0.632.231.422.
Verhandlungen über ein Freihandelsabkommen mit den USA ist das Kooperationsforum für Handel und Investitionen geblieben. $4^{6}$ Diese Einrichtung erlaubt es, dass die Schweiz jederzeit Fragen im $\mathrm{Zu}-$ sammenhang mit der Handels- und Investitionspolitik der USA aufbringen kann, was im besten Fall dazu führt, dass rasch Lösungen gefunden werden können für neu auftretende Herausforderungen in den Wirtschaftsbeziehungen Schweiz - USA. Im Rahmen dieses Kooperationsforums wurde von Seiten der USA die Bereitschaft signalisiert, die Schweiz bzw. die EFTA-Mitgliedstaaten nach Abschluss der Verhandlungen in das TTIP-Abkommen aufzunehmen. 47

14 Weder die Schweiz noch die EU verfügen in den USA derzeit über einen privilegierten Marktzugang. Die USA verfügen über Freihandelsabkommen mit Ländern aus Nord-, Zentral- und Lateinamerika, sowie aus Südostasien, dem Mittleren Osten und Australien.48 Bisher waren Produkte und Dienstleistungen aus der EU daher auf dem amerikanischen Markt mit Schweizer Produkten und Dienstleistungen gleichgestellt. In den vergangenen zwei Jahren haben die USA mehrere plurilaterale Verhandlungen aufgenommen und lanciert. Nebst den Verhandlungen über das TTIP-Abkommen EU USA, laufen Verhandlungen über ein Transpazifisches Freihandelsabkommen

$4 6 \longdiv { \text { Siehe Motion } 0 6 . 3 0 2 2 \text { zur Schaffung des schweize- } }$ risch-amerikanischen Kooperationsforums und $\mathrm{Ab}$ schluss von Wirtschaftsabkommen mit den USA von Ständerat Peter Briner, 7. März 2006.

47 Siehe Burkhardt, Peter, Schweiz hat Chancen auf Big Deal: Die EU und die USA verhandeln über die grösste Freihandelszone der Welt - und signalisieren, dass die Schweiz beitreten darf, Schweiz am Sonntag, 2014.

48 Siehe Office of the United States Trade Representative. 
(Trans-Pacific Partnership, TPP), und über ein plurilaterales Abkommen über Dienstleistungen (Trade in Services Agreement, TiSA), an welchen sowohl die EU wie auch die Schweiz teilnehmen.

Die USA sind der zweitwichtigste Handelspartner der Schweiz. 49 Besonders wichtig ist der amerikanische Markt für die Schweiz im Bereich der Automobilindustrie, der Dienstleistungen und der Chemie-/Pharmaindustrie.50 Auch der Schweizer Export in die USA im Beschaffungswesen ist beachtlich. ${ }^{1}$ Wegen den vergleichbaren Produktionsbedingungen und ähnlichen Standortvorteilen, erhalten Schweizer Produkte und Dienstleistungen im amerikanischen Markt direkte Konkurrenz aus der EU. Wenn folglich die Mitgliedstaaten der EU einen gegenüber den Schweizer Anbietern privilegierten Marktzugang erhalten, trifft dies den Schweizer Export an einer empfindlichen Stelle und hat Auswirkungen auf das Gesamtvolumen der Exporte der Schweiz. Wie die Studie des World Trade Institutes zeigt, hat ein Abseitsstehen auf alle Fälle negative Auswirkungen auf das Exportvolumen, unabhängig von der Ausgestaltung des Abkommens.52 Einzelne Sektoren werden stärker betroffen sein, andere weniger. Voraussichtlich werden in erster Linie die Sektoren in welchen die gegenseitige Anerkennung von Standards von zentraler Bedeutung ist und in welchen heute noch Zölle bestehen, vom TTIP-Abkommen betroffen sein. Andere Sektoren, wie Dienstleistungen, dürften über das TiSA und den

\footnotetext{
49 Bundesamt für Statistik, 2013.

50 Cottier et al., Potential Impacts of a EU-US Free Trade Agreement on the Swiss Economy and External Economic Relations, 2014, S. 21-2.

51 Ibid., S. 87ff.

52 Ibid., S. 38ff.
}

allgemeinen Standard unter dem WTORecht relativ gut abgesichert bleiben. 53

16 Insbesondere in der Maschinenindustrie bestehen in den USA noch Zölle in der Höhe von bis zu 10\% auf einzelne Produkte und Bestandteile.54 In der Pharmaund Chemieindustrie hingegen ist die gegenseitige Anerkennung von Produktionsstandards entscheidend. In beiden Fällen ist eine für das Schweizer Exportvolumen wichtige Industrie betroffen. Falls es der Schweiz nicht gelingen sollte, die negativen Auswirkungen auf die genannten Industrien zu minimieren - z.B. über den autonomen Nachvollzug der Produktionsstandards - bleibt offen, ob die vom Abseitsstehen der Schweiz am stärksten betroffenen Firmen ihre Produktion oder auch ihren Sitz ins Ausland verlegen werden. Entsprechende Äusserungen wurden allerdings bereits vorgebracht. 55

\section{Die Kosten des Abseitsstehens für Kleinstaaten}

17 Eine vertiefte wirtschaftliche Integration zwischen den USA und der EU und ohne die Schweiz hätte für die Schweiz Konsequenzen auf verschiedenen Ebenen. Einige der Folgen eines solchen MegaAbkommens sind einfach zu benennen, während über andere - potentiell gewichtige - zum heutigen Zeitpunkt nur gemutmasst werden kann. Die zugrundeliegende Schwierigkeit für die Schweizer Aussenwirtschaftspolitik - nämlich die Verschiebung der Weiterentwicklung des Handelsrechts in die Verhandlungen über Mega-Abkommen - wird allerdings

53 Ibid., S. 50ff.

54 Siehe auch Francois und Sieber-Gasser, 2014.

55 Bazonline, Bilaterale sind für Novartis „extrem wichtig", 2014. 
auch weiterbestehen, sollte das TTIPAbkommen nicht zustande kommen. 56

Die bisherige Aussenwirtschaftspolitik der Schweiz war stark am Multilateralismus der WTO ausgerichtet. Daneben war die Schweiz in den vergangenen Jahren äusserst aktiv, im Rahmen der EFTA oder auch im Alleingang verschiedene Freihandelsabkommen mit wichtigen Handelspartnern abzuschliessen.57 Diese Strategie ging für die Schweiz gut auf, denn sie konnte starke Partnerländer für Freihandelsabkommen finden und profitierte als kleiner Staat vom Schutz vor den wirtschaftlichen Grossmächten durch die Minimalstandards der WTO. Auch die guten Handelsbeziehungen mit der EU haben den politischen Turbulenzen lange standgehalten und die Schweizer Wirtschaft konnte weitgehend am EU-Binnenmarkt teilnehmen, ohne grössere Wettbewerbsnachteile als Folge der Nicht-Mitgliedschaft in der EU in Kauf nehmen zu müssen.

19 Die Zeiten haben sich wohl geändert. Es ist eher unwahrscheinlich, dass sich die heute 160 Mitgliedstaaten der WTO in absehbarer Zeit auf eine umfassende Weiterentwicklung des internationalen Handelsrechts einigen werden. $5^{8}$ Dies schadet insbesondere kleinen Staaten, wie der Schweiz. Verschiedene Bereiche der Handelsregulierung verlangen heute

$56 \overline{\text { Ähnlich dazu Cottier, Thomas, Paradigmenwechsel }}$ für die Schweizer Aussenwirtschaftspolitik, Gastkommentar zum möglichen Freihandel zwischen der EU und den USA, NZZ, 2013.

57 Seco, Freihandelsabkommen, 2014.

58 Wahrscheinlicher ist, dass die WTO ihre Rolle im globalen Handelssystem neu definieren muss, siehe dazu auch Matsushita, Mitsuo, A View on Future Roles of The WTO: Should There be More Soft Law in The WTO?, Journal of International Economic Law, vol. 17, no. 3, 2014, S. 701-715. nach einer Erweiterung und Anpassung, so z.B. der Dienstleistungshandel, welcher weltweit immer wichtiger wird, dessen internationale Regulierung aber noch in den Kinderschuhen steckt59; aber auch die Bestimmungen über nicht-tarifäre Handelshemmnisse müssen erweitert werden, weil letztere heute viel stärker ins Gewicht fallen, als Zölle6o. Mit der weltweiten Finanzkrise wurde auch deutlich, dass die Finanzmärkte und damit Investitionen besser reguliert werden müssen ${ }^{61}$. Und schliesslich setzt sich das Bewusstsein für neue Aspekte des Welthandels immer mehr durch, so z.B. Handel in Verbindung mit Klimaschutz, mit Menschenrechten, mit Migration und mit Entwicklung62. Weil sich auf multilateraler Ebene in diesen Fragen wenig bewegt, wird auch in den Mega-Abkommen an der Weiterentwicklung dieser Bereiche des Handelsrechts gearbeitet. Dies mag insbesondere auch ein Grund für die Aufnahme von Verhandlungen über Mega-Abkommen sein, denn wem es gelingt, den neuen internationalen Stan-

59 Siehe z.B. Hoekman, Bernard und Mattoo, Aaditya, Services Trade Regulation and Regulatory Reform: Re-Invigorating International Cooperation, World Bank Policy Research Paper No. 5517, World Bank, Washington DC, 2011.

60 Baldwin, Richard, 21st Century Regionalism: Filling the Gap between 21st Century Trade and 20th Century Trade Rules, WTO Staff Working Paper ERSD-2011-08, Geneva, 2011.

61 Aslund, Anders, The World Needs a Multilateral Investment Agreement, Policy Brief No PB13-01, Peterson Institute for International Economics, Washington DC, 2013.

62 Siehe z.B. Horn, Henrik, Mavroidis, Petros C. und Sapir, André, Beyond the WTO? An Anatomy of EU and US Preferential Trade Agreements, The World Economy, vol. 33, nr. 11, 2010, S. 15651588; Stoler, Andrew L., Addressing $21^{\text {st }}$ Century WTO-Plus Issues in the Multilateral Trading System, in: R. Meléndez-Ortiz, C. Bellmann und M. Rodrigues Mendoza (Hrsg.), The Future and the WTO: Confronting the Challenges, ICTSD und SECO, Genf, 2012, S. 42-47, S. 45. 
dard einzuführen, der hat wesentliche Vorteile.

In diesen Fora können wirtschaftliche Grossmächte wie die USA oder die EU ihre gesamte Verhandlungsmacht ausspielen und kleinere Länder so vermehrt in Bedrängnis bringen. Solange sich kleinere Staaten nicht zusammenschliessen um eine kritische Masse zu erreichen (wie dies beispielsweise die EU für die kleinen EU-Mitgliedstaaten darstellt), mögen sie in diesen Verhandlungskonstellationen vermehrt vor der Wahl stehen, entweder die Bedingungen, welche von den grossen Märkten diktiert werden zu akzeptieren, oder aber aussen vor zu bleiben. Hier bietet der Multilateralismus der WTO einen grossen Vorteil für kleinere Staaten, indem dieselben Regeln für alle gelten, es kleineren Staaten möglich ist sich mit anderen zusammenzuschliessen, und letztendlich auch, weil alle wirtschaftlichen Grossmächte am selben Tisch sitzen und damit die Verhandlungsmacht besser verteilt ist. ${ }^{63}$ In dem Sinn ist der momentane Stillstand der Verhandlungen in der WTO für die Schweiz mehr noch als für andere Industrieländer ein Nachteil.

Aber auch die bilateralen Beziehungen zwischen der Schweiz und der EU sind spätestens seit der Abstimmung über die Masseneinwanderungsinitiative im Februar 2014 auf die Probe gestellt.64 Wäh-

63 Dies gilt nur schon wegen dem Konsensprinzip; siehe auch Nguyen, Than, Nguyen, Quyng und Pham, Phong, Decision-Making by Consensus in the WTO, SSRN, 2012; interessant auch Low, Patrick, WTO Decision-Making for the Future, Staff Working Paper, WTO, Geneva, 2011.

64 Bundesverwaltung der Schweizerischen Eidgenossenschaft, Folgen der Annahme der Initiative ,gegen Masseneinwanderung ", 2014. rend möglicherweise die gesamten Bilateralen I auf dem Spiel stehen, sollte die Schweiz ihren Verpflichtungen unter dem Abkommen über die Personenfreizügigkeit nicht mehr nachkommen, ${ }^{65}$ fehlt es schon länger an der nötigen Kohärenz in den handelsrechtlichen Beziehungen Schweiz - EU: die statischen bilateralen Abkommen vermögen nicht Schritt halten mit der schnellen und dynamischen Weiterentwicklung des Europarechts. ${ }^{66}$ Solche letztlich institutionellen Fragen sind auch Gegenstand der TTIP-Verhandlungen. ${ }^{67}$ Es bestehen bereits verschiedene Vorschläge für einen institutionellen Rahmen EU - USA, welcher eine dynamische Weiterentwicklung und Vertiefung der Rechtsharmonisierung gewährleisten soll. 68 Angesichts der aktuellen Herausforderungen in vergleichbaren Fragen zwischen der Schweiz und der EU verbleibt noch offen, inwiefern sich ein institutioneller Rahmen im TTIP-Abkommen auch als Vorlage für

65 Bundesrat, Vorabdruck des Aussenpolitischen Berichts 2014, Bern, 2015, S. 53; Elser, Dominik,

Swiss accept Initiative to „Stop Mass Immigration”: Legal Implications (Part II), Cambridge Journal of International and Comparative Law, 2014; Kaddous, Christine, Rechtsgutachten, Economiesuisse, 2013; Lazowski, Adam, The End of Chocolate BoxStyle Integration? EU-Swiss Relations after the Referendum, CEPS Commentary, 2014.

66 Siehe dazu beispielsweise Cottier, Thomas und Liechti, Rachel, Zwischen Skylla und Charybdis: Die Rezeption des europäischen Wirtschaftsrechts der Schweiz, Europäische Zeitschrift für Wirtschaftsrecht, Schwerpunktheft Schweiz, 2012, S. 841-880, S. 852.

67 European Commission, Trade Cross-Cutting Disciplines and Institutional Provisions, Initial EU Position Paper, 2013, S. 4; Alemanno, Alberto, The Transatlantic Trade and Investment Partnership and the Parliamentary Dimension of Regulatory Cooperation, Directorate-General for External Policies of the Union, European Parliament, 2014.

68 Siehe kürzlich veröffentlichte Verhandlungstexte der EU: Europäische Kommission, Regulatory Cooperation TTIP, 2015. 
die Beziehungen Schweiz - EU herausstellen könnte.

Eine der Konsequenzen des TTIPAbkommens wird sein, dass die darin verhandelten Produktionsstandards und Richtlinien einen globalen Massstab setzen werden: Wenn sich die EU und die USA auf gemeinsame Bestimmungen einigen können, können diese von Drittstaaten nicht ignoriert werden. Eine der möglichen Folgen der NichtMitgliedschaft der Schweiz in der EU und der neuen Verhandlungen über MegaAbkommen ist, dass die Schweiz zusammen mit anderen Kleinstaaten aussenwirtschaftspolitisch immer mehr an den Rand gedrängt werden kann, aus Mangel an wirtschaftlicher und politischer Bedeutung. Wenn wirtschaftliche Grossmächte in Mega-Abkommen entscheiden, können sich Kleinstaaten wie die Schweiz zum sogenannten «autonomen Nachvollzug» gezwungen sehen um eine Marginalisierung zu verhindern.

\section{Neue Herausforderungen in der Aussenwirtschaftspolitik}

Dass die neuen Mega-Abkommen eine bedeutsame Entwicklung im internationalen Handelsrecht darstellen, ist spätestens seit der Aufnahme von Verhandlungen zwischen der EU und den USA in das Bewusstsein der europäischen Öffentlichkeit gerückt und neu auch in der Schweiz zunehmend ein Thema. Wegen der Komplexität solcher Verhandlungen, aber auch generell des internationalen Handelsrechts und wohl auch weil in der Vergangenheit zu wenig über die Entwicklungen im internationalen Handelsrecht informiert worden ist, verbreiten Schlagwörter wie „Hormonfleisch“ und
„Chlorhühnchen“ eine diffuse Angst vor diesen neuen Entwicklungen.

\section{Von Chlorhühnchen und skrupel- losen Investoren}

24 So wird behauptet, dass wegen dem TTIP-Abkommen sogenannte Chlorhühnchen und Hormonfleisch in die Schweiz gelangen würden 69 und in einem vor kurzem erschienenen Politblog wird gesagt, dass durch die Investor-Staat Streitbeilegung Menschenrechte verletzt werden. $7^{70}$ Das sind zum grössten Teil schlicht falsche Behauptungen. Hormonfleisch beispielsweise ist nicht eine neue Erfindung der USA. Die Frage des Exports von Hormonfleisch in die EU wurde bereits in einem WTO Streitbeilegungsverfahren geklärt71 und es wird heute - obwohl das Importverbot nicht vereinbar ist mit WTO-Recht - kein Hormonfleisch nach Europa exportiert. Im Gegenzug gegen das Import-Verbot hat die EU die generellen Kontingente für Fleisch-Importe aus den USA erhöht.72 Ähnliche Verhandlungslösungen sind auch in anderen Bereichen der verarbeiteten Landwirtschaftsprodukte $\mathrm{zu}$ erwarten, so ist es denn höchst unwahrscheinlich, dass je ein sogenanntes Chlorhühnchen in einem europäischen Lebensmittelgeschäft angeboten wird.73

69 Tageswoche.ch, Kommt das Chlor-Hühnchen auch in die Schweiz?, 14. August 2014; WoZ (2014) Das Chlorhuhn ist nicht das Schlimmste, 2014; Grüne, Freihandelsabkommen EU-USA, Faktenblatt, 2014; JUSO, Nein zum Abbau von Demokratie und Service Public - Nein zu TTIP und TISA, Resolution der GL, 2014.

70 Molina, Fabian, Wer schützt uns vor fremden Richtern?, Politblog tamedia, 2014.

71 EC - Hormones.

72 European Commission, EU Commission and Canada reach provisional solution in beef dispute, 2011.

73 Ein Streitbeilegungsverfahren über den Export von Chlorhühnchen in die EU ist hängig bei der WTO 
25 Ähnlich einzuschätzen ist auch die Debatte über die Investor-Staat Streitbeilegung, welche möglicherweise in das TTIP-Abkommen aufgenommen werden könnte: Die Investor-Staat Streitbeilegung wird vehement für seine Intransparenz kritisiert und dafür, dass Schiedsgerichte im Interesse von Investoren Staaten $\mathrm{zu}$ Schadenersatzzahlungen verpflichten können.74 Es bleibt offen, ob und in welcher Form dies tatsächlich geschehen wird, da sich die EU und die USA in dieser Frage bisher uneinig sind.75 Zudem erscheint der Zeitpunkt der Diskussion auch einigermassen erstaunlich, haben doch sämtliche EU Mitgliedstaaten - und ebenso die Schweiz bereits solche Verfahren in Abkommen mit anderen Ländern - inkl. den USA -

(EC - Poultry). Auf Begehren der USA 2009 wird ein Panel eingerichtet. Zu den Hintergründen siehe auch Johnson, Renér, U.S. - EU Poultry Dispute, Congressional Research Service, Washington DC, 2010. Siehe auch von Petersdorff, Winand, Bei uns gibt's heute Chlorhuhn, Frankfurter Allgemeine, 2014; Szigertvari, Andras, Kein Chlorhuhn bei Freihandelspakt, derStandard.at, 2014.

74 Interessanterweise liegt der Fokus der öffentlichen Debatte primär auf dem TTIP, während die Tatsache, dass das CETA (Freihandelsabkommen EUKanada) bereits ein entsprechendes Kapitel fertig verhandelt hat eher im Hintergrund bleibt, siehe z.B. Müller, Christian, CETA: Die Geier warten die Deutschen pennen, infosperber.ch, 2014; Eberhardt, Pia, Investitionsschutz am Scheideweg: TTIP und die Zukunft des globalen Investitionsrechts, Friedrich Ebert Stiftung, Berlin, 2014. Als Reaktion auf den Druck der Öffentlichkeit wurde eine öffentliche Konsultation einberufen, siehe Europäische Kommission, Europäische Kommission startet öffentliche Online-Konsultation über Investorenschutz in Transatlantischer Handels- und Investitionspartnerschaft, Pressemitteilung, 2014; dazu auch Van Harten, Gus, Why Arbitrators not Judges?, Kommentar, 2014.

75 Die USA insistieren bis heute zumindest öffentlich auf ein Investitionsschutzkapitel im TTIP, während sich die EU Kommission unterschiedlich äussert aber tendenziell dagegen ist. Siehe z.B. ContraMagazin. eingeführt. ${ }^{6}$ Es handelt sich dabei also ebenfalls nicht um eine neue Erfindung. Die Kritik an den Schiedsverfahren wird in der Schweizer Öffentlichkeit selten gekontert, dabei gilt es aber Folgendes zu beachten: Thematisch sind InvestorStaat Streitigkeiten immer im internationalen Recht angesiedelt und ein Staatsvertrag dient als rechtliche Grundlage. Das ist folglich ein Rechtsbereich, mit welchem sich die wenigsten Richterinnen und Richter vertieft auseinandersetzen, insbesondere auch, weil solche Streitigkeiten relativ selten vorkommen. Ein Verfahren vor einem spezialisierten Schiedsgericht mag intransparenter sein, aber das daraus resultierende Urteil ist üblicherweise von hoher rechtlicher und inhaltlicher Qualität und deutlich weniger politisch gefärbt als inhaltlich ähnlich gelagerte Gerichtsurteile.77 Wäre dem nicht so, hätte sich das System der Investor-Staat Streitbeilegung kaum in diesem

76

Investitionsschutzabkommen zwischen den USA und EU Mitgliedsländern (Bulgarien, Kroatien, Tschechien, Estland, Lettland, Litauen, Polen, und Rumänien); Investitionsschutzabkommen zwischen EU Mitgliedsländern, siehe Tietje, Christian, Bilateral Investment Treaties Between EU Member States (Intra-EU-BITs) - Challenges in the Multilevel System of Law, Transnational Dispute Management, vol. 10, nr. 2, 2013; Auch die Schweiz hat über 120 Bilaterale Investitionsschutzabkommen abgeschlossen, siehe Staatssekretariat für Wirtschaft SE$\mathrm{CO}$, Liste der Abkommen, Stand 2. November 2014.

$77 \mathrm{Zu}$ den Gründen, die für Schiedsgerichte sprechen, siehe Garcia, Carlos G., All the other dirty little Secrets: Investment Treaties, Latin America, and the Necessary Evil of Investor-State Arbitration, Florida Journal of International Law, vol. 16, 2004, pp. 301-369; Für eine Diskussion der Qualität der Urteile, siehe z.B. Collins, David, Review of 2013 International Centre for Settlement of Investment Disputes (ICSID) Decisions, Manchester Journal of International Economic Law, vol. 10, no. 3, 2013, pp. 423-442. Siehe auch Bubrowski, Helene, Schiedsgerichte sind gerechter, Frankfurter Allgemeine, 2014. 
Ausmass etablieren können: Weltweit sind heute über 2388 solcher Abkommen in Kraft.78

Die Diskussion über eine Weiterentwicklung des Systems der Investor-Staat Streitbeilegung wird bereits seit längerem sowohl in der Wissenschaft, wie auch in der Politik geführt. 79 Generell ist es so, dass den vielseitig geäusserten Bedenken bezüglich Transparenz und Demokratie, sowie Staatssouveränität mit einem entsprechend angepassten Verfahren Rechnung getragen werden kann. So können beispielsweise Regulierungen zum Schutz der Umwelt von der Streitbeilegung ausgenommen werden oder Transparenz kann mit einfachen Mitteln erhöht werden.8o Der Protest gegen die Investor-Staat Streitbeilegung primär in Europa ist letztendlich paradox: Die meisten Streitbeilegungsverfahren weltweit werden von Investoren aus EU Mitgliedstaaten initiiert (53\%), gefolgt von den USA (22\%). Das System wird also rege genutzt, wenn es um die Interessen der eigenen Investoren geht, während aussereuropäischen Investoren nicht dieselben Möglichkeiten zugestanden wer-

UNCTAD, Investment Policy Hub, 2015.

Siehe beispielsweise Chung, Olivia, The Lopsided International Investment Law Regime and Its Effect on the Future of Investor-State Arbitration, Virginia Journal of International Law, vol. 47, no. 4, 2006, S. 953-976; Laird, Ian and Askew, Rebecca, Finality versus Consistency: Does Investor-State Arbitration need an Appellate System?, The Journal of Appellate Practice and Process, vol. 7, no. 2, 2005, S. 285-302; oder Henckels, Caroline, Indirect Expropriation and the Right to Regulate: Revisiting Proportionality Analysis and the Standard of Review in Investor-State Arbitration, Journal of International Economic Law, vol. 15, no. 1, 2012, pp. 223-255.

80 Magraw, Daniel und Amerasinghe, Niranjali, Transparency and Public Participation in InvestorState Arbitration, ILSA Journal of International and Comparative Law, vol. 15, nr. 2, 2008, pp. 337-360. den. Diese Haltung ist problematisch, weil der Schutz der eigenen Investoren letztendlich von zwischenstaatlicher Reziprozität abhängig ist. ${ }^{81}$

\section{Der eigentliche Kern der Heraus- forderung}

27 Während die Verhandlungen über MegaAbkommen wie das TTIP das Handelsrecht - aber auch die Gesellschaft - vor neue Herausforderungen stellen, so ist die emotionale Kritik verbunden mit der Streitbeilegung und den Chlorhühnchen verfehlt. Beides sind Facetten einer grösseren Dynamik und eine isolierte Diskussion führt kaum zu befriedigenden Lösungen. Ganz grundlegend drängt sich die Frage auf, wie in einer modernen und demokratischen Gesellschaft die Bevölkerung sowie Kleinere und Mittlere Unternehmen (KMUs) in den Verhandlungsprozess miteinbezogen werden können. Im Gegensatz zu früher betreffen Handelsabkommen wegen der Globalisierung und ihrem Umfang uns alle in immer mehr Bereichen unseres Alltags. Insbesondere wenn neue Rechtsbereiche wie Klimaschutz, Menschenrechte oder die Regulierung des Internets in das Handelsrecht aufgenommen werden, sind nicht mehr nur primär Exportunternehmen direkt von einem Handelsabkommen betroffen. Gerade in einer Demokratie vermag dies den Bedarf an verstärkter Mitsprache und demokratischer Legitimierung rechtfertigen. Angesichts der diversen Kampagnen gegen das TTIP sowohl im benachbarten europäischen Ausland $^{82}$, wie auch im Inland ${ }^{83}$, wird die

81 Polanco, Rodrigo, The No of Tokyo Revisited: Or How Developed Countries Learned to Start Worrying and Love the Calvo Doctrine, ICSID Review, vol. 29, nr. 4, 2014, pp. 1-23, p. 20.

82 Siehe beispielsweise Self-organised European Citi- 
europäische aber auch die schweizerische Handelspolitik nicht umhin kommen, in Zukunft die Mitsprache der Zivilgesellschaft und der KMUs institutionell vorzusehen. Verschiedene entsprechende Vorstösse bestehen bereits, aber bis zum heutigen Zeitpunkt wurde noch wenig dafür unternommen, den Verhandlungsprozess nachhaltig transparenter und demokratischer zu gestalten. $84 \mathrm{Um}$ das TTIP überhaupt in Kraft setzen zu können, stehen Regierungen in Europa vor der Herausforderung, neue Wege der Verhandlungsführung einzuschlagen, welche der Öffentlichkeit ein Recht auf Anhörung und Information einräumen. Erste Ansätze sind im öffentlichen Konsultationsverfahren zur Investor-Staat Streitbeilegung erkennbar, oder auch in

zen's Initiative, Stop-TTIP; StopTTIP UK; Campact, Stoppt TTIP; Attac, Freihandelsfalle TTIP; The Greens in the European Parliament, TTIP Leak, ttippen.se, TTIP Beware what lies beneath; EurActiv.com, Anti-TTIP demonstrations seize European capitals; RT Network, \#NoTTIP: Mass protests slam US-EU trade deal as 'corporate power grab'; Heidenheimer Zeitung, Protestbewegung will "Trojanisches Pferd" stoppen.

83 Siehe beispielsweise Aktive Schweiz, Stop TTIP; WOZ, Gefährliches Andocken; Balthasar Glättli, TTIP gefährdet Gesellschaft und Umwelt; Politnetz.ch, EU, USA, WTO, NATO, NSA, TTIP, TiSA: Alles ",Hans was Heiri"?; Komitee „Stop TisA“, Gegen die Diktatur der Multinationalen Unternehmen; Grüne, Kein Freihandel zu Lasten von Umwelt und Gesellschaft.

84 Sander, Matthias, Demokratie-Aktivisten verklagen die EU, NZZ, 2014; Eberhardt, Pia, Redlin, Blair und Toubeau, Cecile, Verkaufte Demokratie, corporateeurope.org, 2014; Spiegel Online, Protest gegen Abkommen: EU-Kommission lehnt Bürgerinitiative von TTIP-Gegnern ab, 2014; Williams, Lee, What is TTIP? And six reasons why the answer should scare you, The Independent, 2014; Gerstetter, Christiane et al., Regulatory Cooperation under TTIP - a Risk for Democracy and National Regulation?, Heinrich Böll Stiftung, Berlin, 2014; Crouch, Colin, Democracy at a TTIPing point: Seizing a Slim Chance to Reassert Democratic Sovereignty in Europe, Juncture, vol. 21, no. 3, 2014, pp. 176-181. der Veröffentlichung einzelner Verhandlungstexte. ${ }^{85}$

28 Auch die Schweiz braucht neue Ansätze in der Handelspolitik und in der Verhandlungsstrategie um eine Marginalisierung im Weltmarkt zu verhindern. Der Trend zu Mega-Abkommen und zur Weiterentwicklung des Handelsrechts wird auch für die Schweiz spürbar werden und eine Reaktion der Schweizer Handelspolitik verlangen. Damit die Schweiz allerdings reagieren kann, sollte die Öffentlichkeit bereits heute über die Entwicklungen und möglichen Optionen informiert werden. Denn der naheliegendste Ansatz zur Verhinderung einer Marginalisierung ist der Anschluss an MegaAbkommen, sei dies in der Form eines Andockens oder auch in der Form von einer Mitgliedschaft in einer bestehenden oder neuen Staatengemeinschaft. Eine solche Reaktion kann allerdings nur proaktiv stattfinden, wenn diese von der Öffentlichkeit mitgetragen wird. Falsche Behauptungen über Chlorhühnchen und skrupellose Investoren sollten daher nicht widerspruchslos toleriert werden.

$85 \overline{\text { European Commission, Online public consultation }}$ on investment protection and investor-to-state dispute settlement (ISDS) in the Transatlantic Trade and Investment Partnership Agreement (TTIP), 2014; eine Einschätzung der Public Consultation beispielsweise von Weiniger, Matthew und Naish, Vanessa, The Future of Investor-State Arbitration, Arbitration Notes, Lexocology.com, 2014;

Euractive.com, Commission launches transparency initiative for TTIP and lobbying, 2014; Delegation of the European Union to the United

States, European Commission publishes TTIP Legal Texts as Part of Transparency Initiative, 2015; European Commission, Now Online - EU negotiating Texts in TTIP, 2015. 
IV. Die Neue Komplexität als Chance

29 Sofern der Trend zu Mega-Abkommen Bestand hat, werden die nächsten Jahre der globalen Handelspolitik davon dominiert. ${ }^{86}$ Mega-Abkommen wie das TTIP stellen aber nicht nur eine Bedrohung der Demokratie dar sondern sind auch eine Chance für die Weiterentwicklung des Handelsrechts. Anstatt wegen kritischer Einzelaspekte den ganzen Prozess zu boykottieren, wäre es zielführender, konkrete Vorschläge für die Verbesserung und Weiterentwicklung des Handelsrechts vorzubringen. Denn können sich die EU und die USA beispielsweise auf eine Weiterentwicklung der InvestorStaat Streitbeilegung im Sinne von mehr Transparenz und Legitimation einigen, dann ist dies nicht nur für Europa eine Errungenschaft sondern auch für die Schweiz. Oder anstatt wegen Chlorhühnchen das ganze Abkommen zu bekämpfen, könnte beispielsweise der Einbezug von Standards zur ethischen Fleischproduktion gefordert werden. Die Diskussion über das TTIP würde dadurch komplexer, aber sie könnte dazu beitragen, dass das Potential der neuen MegaAbkommen zur Einführung neuer Standards im Interesse der globalen Gemeinschaft wahrgenommen werden kann.

Letztlich steht die Frage, welche Art von Wirtschaftswachstum wir in Zukunft wollen auch in direktem Zusammenhang mit der Form der Freihandelsabkommen, welche wir abschliessen. Angesichts der stetig wachsenden Weltbevölkerung kann es sich die Welt nicht leisten, ganz auf Wirtschaftswachstum zu verzichten.
Europa ist insbesondere auch auf Wirtschaftswachstum angewiesen, angesichts seiner stetig alternden Bevölkerung. Die Art des Wirtschaftswachstums hingegen hängt insbesondere auch von den internationalen Handelsbeziehungen, dem globalen Wettbewerb und damit von Handelsabkommen und vom Handelsrecht ab. Die neuen Mega-Abkommen bieten in dieser Frage eine grossartige Möglichkeit, grundlegende Fragen aufzugreifen und an neuen Lösungen zu arbeiten für ein Handelsrecht, das den Herausforderungen des 21. Jahrhunderts gerecht wird.

86 Interessant dazu, Hoekman, Bernard, Supply Chains, Mega-Regionals and Multilateralism: A Road Map for the WTO, CEPR Press, London, 2014. 